\title{
Combination of Lactobacillus helveticus R0052 and Bifidobacterium longum $R 0175$ reduces post-myocardial infarction depression symptoms and restores intestinal permeability in a rat model
}

\author{
Jessica Arseneault-Bréard ${ }^{1,2}$, Isabelle Rondeau ${ }^{1,2}$, Kim Gilbert ${ }^{1,2}$, Stéphanie-Anne Girard ${ }^{1,2}$, \\ Thomas A. Tompkins ${ }^{3}$, Roger Godbout ${ }^{1,4}$ and Guy Rousseau ${ }^{1,2 *}$ \\ ${ }^{1}$ Centre de biomédecine, Hôpital du Sacré-Cour de Montréal, 5400 Boulevard, Gouin Ouest, Montréal, QC, \\ Canada H4J 1 C5 \\ ${ }^{2}$ Department of Pharmacology, Université de Montréal, Montréal, QC, Canada H3C 3J7 \\ ${ }^{3}$ Institut Rosell-Lallemand Inc., Montréal, QC, Canada H4P 2R2 \\ ${ }^{4}$ Department of Psychiatry, Université de Montréal, Montréal, QC, Canada H3C 3J7 \\ (Submitted 23 March 2011 - Final revision received 3 August 2011 - Accepted 16 August 2011 - First published online 21 September 2011)
}

\section{Abstract}

Myocardial infarction (MI) in rats is accompanied by apoptosis in the limbic system and a behavioural syndrome similar to models of depression. We have already shown that probiotics can reduce post-MI apoptosis and designed the present study to determine if probiotics can also prevent post-MI depressive behaviour. We also tested the hypothesis that probiotics achieve their central effects through changes in the intestinal barrier. MI was induced in anaesthetised rats via 40-min transient occlusion of the left anterior coronary artery. Sham rats underwent the same surgical procedure without actual coronary occlusion. For $7 \mathrm{~d}$ before MI and between the seventh post-MI day and euthanasia, half the MI and sham rats were given one billion live bacterial cells of Lactobacillus helveticus R0052 and Bifidobacterium longum R0175 per d dissolved in water, while the remaining animals received only the vehicle (maltodextrin). Depressive behaviour was evaluated 2 weeks post-MI in social interaction, forced swimming and passive avoidance step-down tests. Intestinal permeability was evaluated by oral administration with fluorescein isothiocyanate-dextran, $4 \mathrm{~h}$ before euthanasia. MI rats displayed less social interaction and impaired performance in the forced swimming and passive avoidance step-down tests compared to the sham controls $(P<0.05)$. Probiotics reversed the behavioural effects of MI $(P<0.05)$, but did not alter the behaviour of sham rats. Intestinal permeability was increased in MI rats and reversed by probiotics. In conclusion, L. helveticus R0052 and B. longum R0175 combination interferes with the development of post-MI depressive behaviour and restores intestinal barrier integrity in MI rats.

Key words: Probiotics: Myocardial infarction: Depression: Intestinal barrier

After myocardial infarction (MI), $65 \%$ of patients present depressive symptoms ${ }^{(1)}$, and about $20 \%$ of them incur major depression $^{(1,2)}$. Evidence of a poor prognosis is particularly strong in patients with such symptoms ${ }^{(3-6)}$ : the risk of cardiac death within 6 months after acute MI is approximately four times greater in patients with depression compared to nondepressed control subjects ${ }^{(5)}$. The mechanism is still hypothetical, but it has been postulated that the inflammatory state observed after MI is responsible for post-MI depression ${ }^{(7)}$. Elevation of pro-inflammatory cytokine levels ${ }^{(8,9)}$ has been documented after myocardial ischaemia, and their attenuation is correlated with depressive behaviour reduction ${ }^{(10)}$.

Our previous study indicated that MI rats present symptoms that are similar to human depression. MI rats drink significantly less sucrose and remain more immobile in the forced swimming test, indicating a state of anhedonia and behavioural despair, respectively. These behavioural signs are blocked by the tricyclic anti-depressant, desipramine, the selective serotonin reuptake inhibitor, sertraline ${ }^{(11,12)}$, and the cytokine synthesis inhibitor pentoxifylline ${ }^{(13)}$.

The present experimental model of post-MI depression manifests an early wave of apoptosis, with a peak at $3 \mathrm{~d}$ after MI, in the hippocampus and amygdala regions of the limbic system involved in the pathophysiology of depression $^{(13,14)}$. We have demonstrated that this apoptosis can be blocked by antidepressant ${ }^{(12)}$ or anti-inflammatory treatments $^{(13,15)}$, indicating a link between inflammation, apoptosis and depression. We have also shown that the

Abbreviations: AR, area at risk; FITC, fluorescein isothiocyanate; MI, myocardial infarction.

*Corresponding author: Dr G. Rousseau, fax +1 514338 2694, email guy.rousseau@umontreal.ca 
probiotics Lactobacillus belveticus and Bifidobacterium longum can reverse MI-induced apoptosis in the limbic system $^{(16)}$ by a mechanism that could include a reduction of pro-inflammatory cytokines. It has been observed that $L$. belveticus $R 0052$ reduces IL- $1 \beta$ and IL- 6 , and not significantly TNF- $\alpha^{(17)}$, whereas B. longum R0175 can reduce IL-8 and TNF- $\alpha^{(18)}$, indicating that each strain seems to have a specific effect on pro-inflammatory cytokine synthesis. In the present experiment, we observe that pro-inflammatory cytokine concentrations are elevated and could contribute to the depression-like symptoms ${ }^{(8,19)}$. Owing to the fact that probiotics seem to have specific effects on pro-inflammatory cytokine synthesis, we decided to use the combination of both strains in our experiments. Moreover, previous clinical and animal trials have shown these two strains to be effective in combination, and there is no indication that individual probiotic microbes have this impact on stress ${ }^{(20,21)}$.

We have observed that MI induces perturbation of the blood-brain barrier ${ }^{(22)}$ by an unknown mechanism. We suspect that other barriers, such as the intestinal system, could be affected as has been demonstrated with proinflammatory cytokines ${ }^{(23)}$. Indeed, pro-inflammatory cytokines open tight junction, increase paracellular permeability and affect the epithelial barrier integrity ${ }^{(23,24)}$. Probiotics, such as L. belveticus R0052, may protect the epithelial cells and thus can be helpful to maintain the intestinal barrier integrity $^{(25,26)}$. In the present experiment, we investigated whether probiotics modulate intestinal barrier permeability in the present post-MI model, which could have an effect on circulating pro-inflammatory cytokines ${ }^{\text {(23) }}$.

The aim of the present study was to test if probiotics can reverse the behavioural syndrome induced by MI. As an anti-apoptotic outcome may be achieved through an increase in the anti-/pro-inflammatory cytokine ratio ${ }^{(27,28)}$ and changes in the intestinal barrier ${ }^{(23,29)}$, another objective was to assess intestinal permeability before and after treatment with probiotics.

\section{Materials and methods}

\section{Ethics statement}

These experiments conformed to animal care guidelines published by the Canadian Council on Animal Care, and the procedures performed were approved by the local Animal Care Committee of Hôpital du Sacré-Coeur de Montréal.

\section{Experimental groups (animals and housing)}

A total of forty 12-week-old Sprague-Dawley rats (Charles River Canada, St-Constant, QC, Canada), weighing between 325 and $350 \mathrm{~g}$ at the beginning of the experiments, were housed individually under constant conditions (temperature: $21-22^{\circ} \mathrm{C}$; humidity: $40-50 \%$ ) and maintained on a $12 \mathrm{~h}$ light $-12 \mathrm{~h}$ dark cycle, with lights on at 08.00 hours. Chow pellets (5075-US Charles River Rodent; Charles River Canada) and tap water were available ad libitum throughout the study. All animals were acclimatised to the housing conditions for
$3 \mathrm{~d}$ after delivery by the supplier before they were randomly distributed among one of the four groups: MI rats treated with probiotics or vehicle and controls treated with probiotics or vehicle.

\section{Probiotics treatment}

The commercial probiotics given were a combination of L. helveticus R0052 and B. longum R0175 (Probio'Stick, provided by Institut Rosell-Lallemand, Montréal, QC, Canada). They were administered by dissolving freeze-dried cultures or vehicle (maltodextrin) in $200 \mathrm{ml}$ of tap water. For $7 \mathrm{~d}$ before MI and between the seventh post-MI day and euthanasia, half of the MI and sham rats had access to one billion live bacterial cells of L. helveticus R0052 and B. longum R0175 per $\mathrm{d}$ dissolved in water, while the remaining animals received only the vehicle. The drinking solution was prepared every 2nd day for the duration of the experiments. Water intake was monitored throughout to ensure that enough bacteria were ingested. This mode of administration was chosen as compared to oral administration to reduce handling stress, which may influence the overall outcome of the study.

\section{In vivo surgical procedure}

The animals were anaesthetised by intramuscular ketamine or xylazine ( 50 and $5 \mathrm{mg} / \mathrm{kg}$, respectively) injection. They were subsequently intubated and anaesthesia was maintained with isoflurane (1\%) ventilation. Electrocardiography was undertaken and heart rate monitored throughout the procedure with electrodes placed on their paws. A left thoracotomy was performed at the fifth intercostal space, permitting occlusion of the left anterior descending coronary artery with 4-0 silk suture (Syneture; Covidien, Mansfield, MA, USA). Ischaemia was confirmed by alteration of the electrocardiographic S-T waves and myocardial surface cyanosis. After $40 \mathrm{~min}$ of ischaemia, the suture was removed, permitting myocardial tissue reperfusion. After the thorax was closed with 2-0 (ribs and muscle) and 3-0 (skin) silk sutures (Syneture; Covidien), the animals were injected with antibiotic (15000 IU penicillin G; Duplocillin LA, Intervet Canada Limited, Whitby, ON, Canada) intramuscularly and analgesic $(0.05 \mathrm{mg} / \mathrm{kg}$ buprenorphine; Schering-Plough Limited, Hertfordshire, UK) subcutaneously before being returned to their respective cages. They received a second analgesic injection $(0.05 \mathrm{mg} / \mathrm{kg}$ buprenorphine) in the morning after surgery.

\section{Behavioural measures}

The tests employed were selected on the basis of their validity regarding behavioural depression syndrome. All tests were conducted individually, in the morning, starting $9 \mathrm{~d}$ after surgery. Social interaction was evaluated on day 9, the forced swimming test was performed on days 14 and 15 and the passive avoidance step-down test on day 16. All animals were killed on day 17. 


\section{Social interaction test}

One pair of rats, which received the same diet (either probiotics or vehicle), was placed together in a cage for $10 \mathrm{~min}$, between 09.00 and 11.00 hours. During this period, two observers blinded to the experimental condition each observed one animal, and the following dependent measures were computed: duration and number of interactions with the other rat, number of grooming events and number of rearings.

\section{Forced swimming test}

Rats were placed individually in a transparent $25-\mathrm{cm}$ diameter pool filled to a depth of $30 \mathrm{~cm}$ with $22-25^{\circ} \mathrm{C}$ water, with no possible escape. Two identical chronometers were needed to time the immobile and escape trial periods of the animals. The test was conducted on $2 \mathrm{~d}$ : day 1 comprised $15 \mathrm{~min}$ of habituation and day 2 entailed the actual 5 min test. On the 2nd day, immobility time was translated into depressive symptoms by comparison to the controls. Groups of MI and control rats were tested between 09.00 and 11.00 hours.

\section{Passive avoidance step-down test}

Rats were placed individually in the passive avoidance test chamber $\left(14 \times 23 \mathrm{~cm}^{2}\right)$ on one platform $\left(14 \times 19 \mathrm{~cm}^{2}\right)$ higher than another $\left(14 \times 14 \mathrm{~cm}^{2}\right)$; to reach the test criterion, they needed to stay on this platform for $60 \mathrm{~s}$, over three consecutive trials (inter-trial interval $=30 \mathrm{~s}$ ). If they stepped down onto the second platform $(2.5 \mathrm{~cm}$ lower $)$, resting all four paws on its grid floor, they received a mild shock to the feet $(1 \mathrm{~s}, 8 \mathrm{~mA})$. The test was started between 13.00 and 15.00 hours. The number of trials needed to reach the test criterion and the number needed to learn the test were noted. Groups of MI and control rats were tested $1 \mathrm{~d}$ before they were killed.

\section{Fluorescein isothiocyanate-dextran assessment}

The animals were starved the night before euthanasia. At $4 \mathrm{~h}$ before being killed, they received fluorescein isothiocyanate (FITC)-dextran diluted in water $(60 \mathrm{mg} / 100 \mathrm{~g}$ body weight) by oral administration. After killing, blood was collected and immediately centrifuged at $3000 \mathrm{~g}$ at $4^{\circ} \mathrm{C}$ for $15 \mathrm{~min}$. Plasma was obtained and the sample $(50 \mu \mathrm{l}$ in triplicate) was deposited in a ninety-six-well plate for quantification against a standard curve prepared from stock solution. Measurements were performed on a Cary Eclipse spectrophotometer (Varian, Mississauga, ON, Canada), with excitation wavelength at $350 \mathrm{~nm}$ and emission at $515 \mathrm{~nm}$.

\section{Infarct size}

After $17 \mathrm{~d}$ of reperfusion, the rats were restrained in a cone bag and decapitated rapidly. Blood was collected for plasma IL-1 $\beta$ and FITC-dextran measurements. The heart was removed and the left anterior descending coronary artery was occluded at the same site to quantify the area at risk
(AR) with Evans blue (0.5\%) infusion through retrograde perfusion into the aorta. The heart was then frozen at $-80^{\circ} \mathrm{C}$ for $5 \mathrm{~min}$ and sliced into four transverse sections of $2 \mathrm{~mm}$. Each section was incubated for $5 \mathrm{~min}$ at $37^{\circ} \mathrm{C}$ in a triphenyltetrazolium chloride (1\%, $\mathrm{pH} 7 \cdot 4)$ solution to distinguish the area of necrosis (I) from the AR. MI was expressed as a percentage of necrosis (I) of the AR $((I / \mathrm{AR}) \times 100)$. In addition, the AR was expressed as a percentage of left ventricle area.

\section{IL-1 $\beta$ measurement}

Blood was sampled at the onset of euthanasia and centrifuged for $15 \mathrm{~min}$ at $3000 \mathrm{~g}$. Plasma was then obtained and frozen at $-80^{\circ} \mathrm{C}$ until ELISA. Plasma IL- $1 \beta$ concentrations were quantified with commercial kits from R\&D Systems Inc. (Minneapolis, MN, USA), according to the manufacturer's specifications.

\section{Statistical analyses}

The results are expressed as mean values and their standard errors. MI size and AR were compared by Student's $t$ test. Factorial ANOVA designs $(2 \times 2)$ were adopted, with MI and probiotics as factors. Simple effects were estimated according to Fields ${ }^{(30)}$ whenever an interaction between factors was significant; otherwise, only main effects were reported. In the passive avoidance step-down test, the numbers of trials were evaluated by Kruskal-Wallis tests, followed by Mann-Whitney $U$ tests, with Bonferroni correction for the number of comparisons. An $\alpha$ level of 0.05 was considered significant.

\section{Results}

Infarct size

The AR, expressed as a percentage of left ventricle area, was similar in both groups and represented $45-55 \%$ of the left ventricle. After $40 \mathrm{~min}$ of ischaemia and $17 \mathrm{~d}$ of reperfusion, MI size was similar between groups (Fig. 1) and represented $35-40 \%$ of the AR.

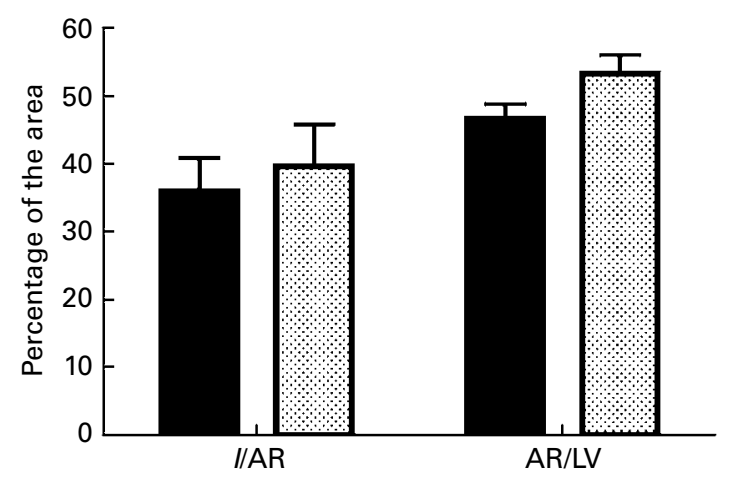

Fig. 1. Myocardial infarct size $(I)$, expressed as a percentage of the area at risk (AR), shows no difference between groups. The AR, expressed as a percentage of the left ventricle (LV), is similar between groups; $n 9$ per group. $\square$, Myocardial infarction (MI) + vehicle; 㘣, MI + probiotics. 


\section{Social interaction}

There was a significant interaction effect between MI and probiotics $(F(1,24)=4.80, P<0 \cdot 05$; Fig. 2$)$; analysis of simple effects indicated that probiotics did not influence interaction time in the sham controls but increased it in MI rats $(F(1,25)=5.92 ; P<0.05)$. No differences were detected in the number of rearings and groomings (data not shown).

\section{Forced swimming test}

We observed significant interaction between factors (MI and probiotics $)$ in swimming time $(F(1,31)=5.90, P<0.05)$ and escape time $(F(1,31)=4.83 ; P<0.05 ;$ Fig. 3$)$. Analysis of simple effects revealed lower values at baseline in the MI groups and improved swimming time $(F(1,32)=10 \cdot 13$, $P<0.05)$ and escape time $(F(1,32)=6.47, P<0.05)$ with treatment. No differences were apparent in the sham groups. Immobility time showed a significant main effect on probiotics $(F(1,31)=5 \cdot 60, P<0.05)$ and $\mathrm{MI}(F(1,32)=10 \cdot 05, P<0 \cdot 05)$.

\section{Passive avoidance step-down test}

We noted significant interaction between MI and probiotics regarding time to learn the test $(F(1,30)=5 \cdot 45, P<0 \cdot 05)$; analysis of simple effects indicated that probiotics decreased time to learning the test in $\mathrm{MI}$ rats $(F(1,31)=4 \cdot 12 ; P=0.05)$ but not in sham rats (Fig. 4). The main effects (MI or probiotics) were NS. The number of trials was significantly different between groups $\left(\chi^{2}=8.475, \quad \mathrm{df}=3 ; \quad P=0.037\right)$ and significantly higher in the MI $v$. the sham group $(Z=-2 \cdot 847 ; P=0.004)$.

\section{Plasma IL-1 $\beta$ concentrations}

No significant treatment $\times$ MI interactions were evident. A main probiotics effect was evident, with probiotics significantly reducing plasma IL- $1 \beta$ concentrations compared to vehicle $(F(1,18)=5 \cdot 70, P<0.05$; Fig. 5$)$. No significant MI main effect was observed.

\section{Intestinal barrier permeability}

We noted significant interaction between $\mathrm{MI}$ and treatment on FITC-dextran concentrations $(F(1,16)=8.60, \quad P<0.05$; Fig. 6). Analysis of simple effects disclosed that FITC-dextran

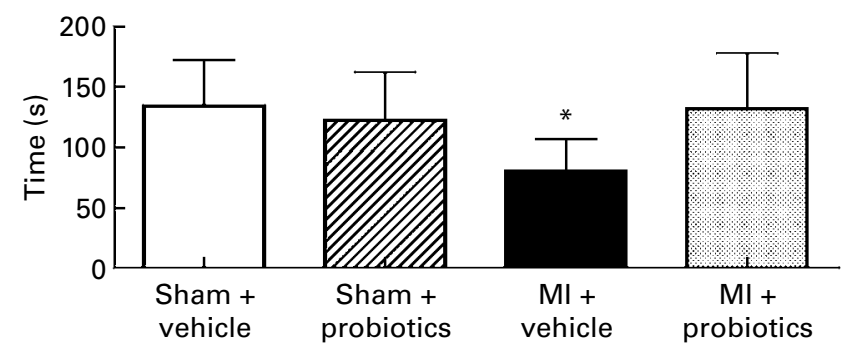

Fig. 2. Social interaction between animals. The data indicate that myocardial infarction (MI)-vehicle rats interact socially less than the other groups. Time is expressed in s; $n$ 6-8 per group. ${ }^{*}$ Values were significantly different for Ml-vehicle $v$. MI-probiotics $(P<0.05)$.
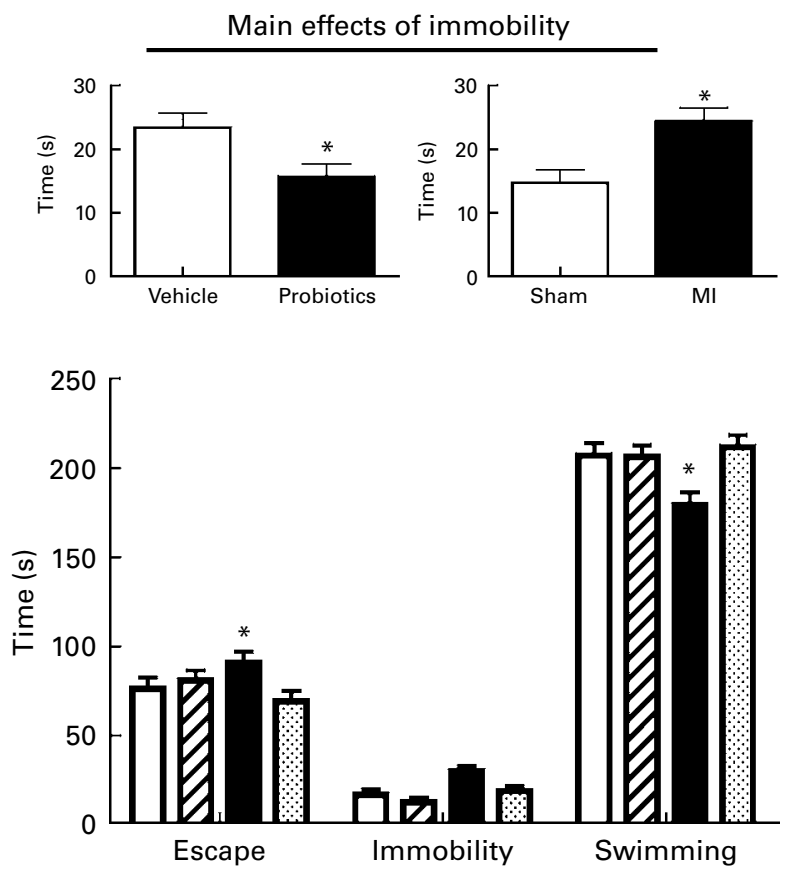

Fig. 3. Performance of rats from the four different groups in the forced swimming test. Escape and swimming time is less in the myocardial infarction (MI)-vehicle compared to the other groups. No interaction was found for the immobility parameter. However, the main effects (inset) indicate that probiotics decrease immobility time, whereas MI rats show more immobility than the vehicle group; $n$ 8-10 per group. * Values were significantly different for MI-vehicle $v$. MI-probiotics $(P<0.05)$. Inset: *Values were significantly different between groups $(P<0.05) . \square$, Sham + vehicle; $\square$, sham + probiotics; $\mathrm{MI}+$ vehicle; 图, $\mathrm{MI}+$ probiotics.

concentrations were significantly increased in $\mathrm{MI}$ rats treated with vehicle compared to $\mathrm{MI}$ rats given probiotics $(F(1,17)=14.92 ; \quad P<0.05)$. No significant effects were observed in sham controls.

\section{Discussion}

We have previously shown that our model of post-MI depression replicates some of the behavioural characteristics of human depression, including anhedonia (sucrose preference test) and behavioural despair (forced swimming test ${ }^{(12)}$. The results obtained in the present experiments constitute further converging evidence that post-MI syndrome is indeed associated with a behavioural repertoire similar to other rat models of depression, and extend behavioural markers to impaired social behaviour (social interaction test) and processing of emotional memory (passive avoidance step-down test).

The present study demonstrates that a probiotic-enriched diet with L. helveticus and B. longum can prevent behavioural despair, abnormal social behaviour and blighted processing of emotional memory. We decided to administer probiotics before MI, as reported previously, to determine if the beneficial effect that we observed on apoptosis in the limbic system $^{(16)}$ is reflected in depressive behaviour. We also know that the administration of probiotics before MI has no influence on infarct size, reducing potential bias in 

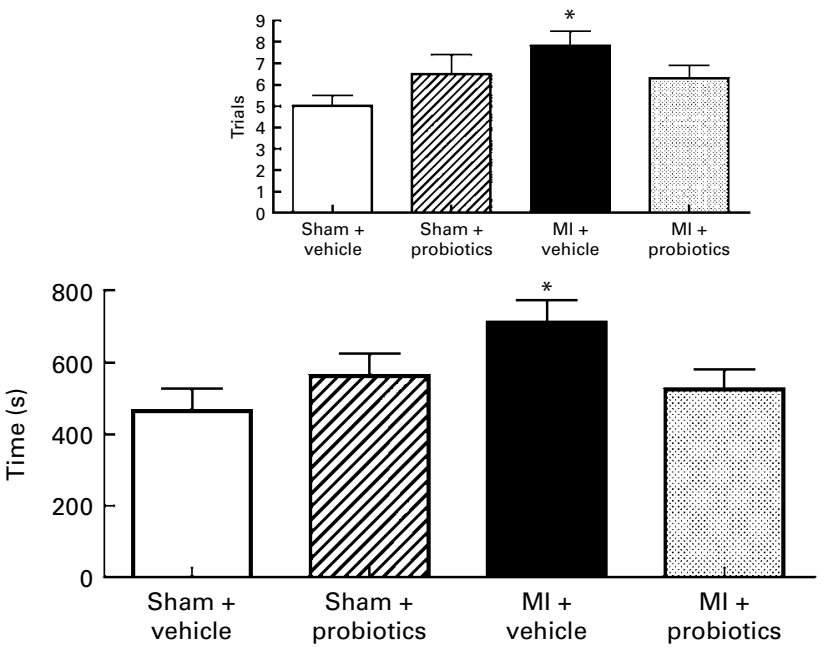

Fig. 4. In the passive avoidance step-down test, myocardial infarction (MI)vehicle rats take more time to succeed in the test than the other groups $(P<0.05)$. Inset: The number of trials is significantly higher in the MI-vehicle than in the other groups; $n$ 8-10 per group. *Values were significantly different for MI-vehicle $v$. MI-probiotics $(P<0.05)$.

performance in the behavioural tests. However, after the surgical procedures, antibiotics were administered to all animals, and to avoid any benefit of probiotics on the potential action of antibiotics, we decide to stop the probiotics for the first $7 \mathrm{~d}$ of reperfusion.

Although the mechanistic relationship between MI and depression is still hypothetical, we postulate that inflammation is probably a key element. It has indeed been documented that pro-inflammatory cytokines can induce depression. For example, IL-1 $\beta$ and TNF- $\alpha$ injection can elicit behavioural changes modelling symptoms of depression, such as decreased locomotion, diminished exploration of the environment, reduced food and water intake, dwindling social interactions and impaired learning as well as memory ${ }^{(31,32)}$. IL-1 $\beta$ and TNF- $\alpha$ are also associated in animals with neuroendocrine
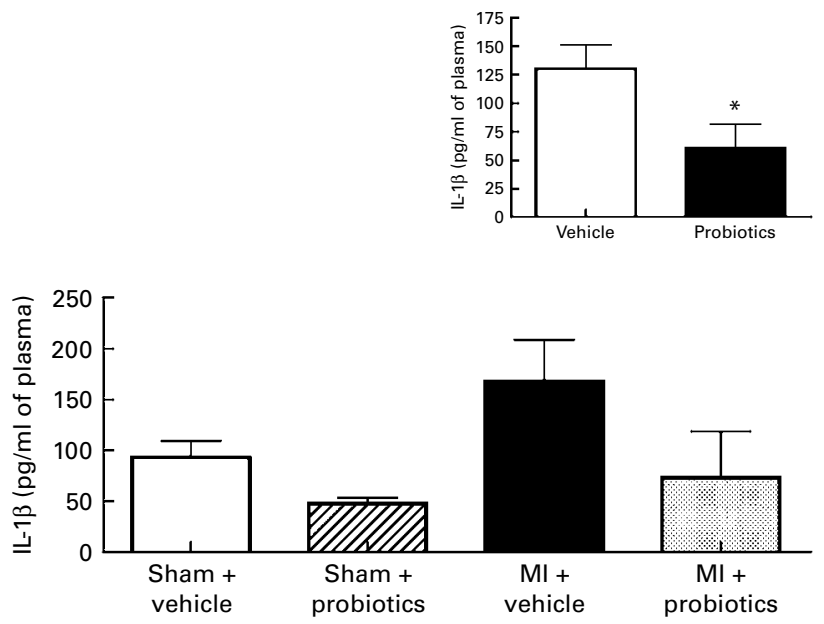

Fig. 5. Probiotics-treated rats present less plasma IL-1 $\beta$ concentrations than vehicle controls (main effect presented in the inset). No other difference was detected between groups; $n$ 5-6 per group. * Values were significantly different for vehicle $v$. probiotics $(P<0.05)$. MI, myocardial infarction.

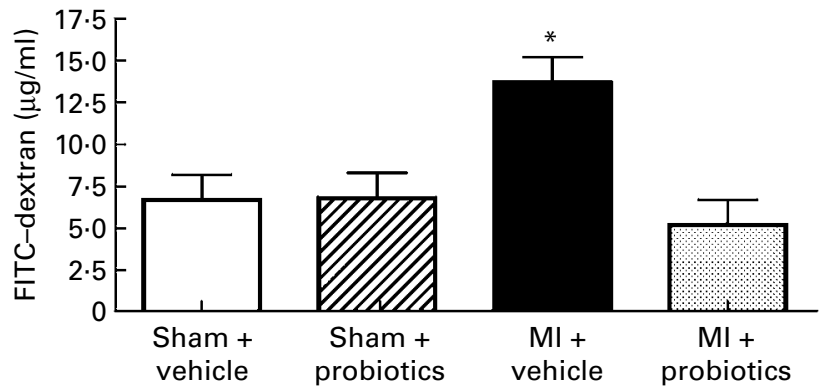

Fig. 6. Fluorescein isothiocyanate (FITC)-dextran concentrations measured in $\mu \mathrm{g} / \mathrm{ml}$ of plasma before euthanasia. The data indicate that FITC-dextran level is significantly higher in the myocardial infarction (MI)-vehicle group than in the other groups; $n 5$ per group. * Values were significantly different for Ml-vehicle $v$. Ml-probiotics $(P<0.05)$.

changes similar to those in depressed humans and responsible for the stress response at the hypothalamic-pituitary-adrenal axis level ${ }^{(33)}$. In addition, there is evidence that antidepressants antagonise the action of cytokines in the brain (for a review see Castanon et al. ${ }^{(34)}$ ), although the mechanism has not yet been elucidated. We believe that the therapeutic effects of $L$. helveticus and B. longum on behavioural signs of depression also involve pro-inflammatory cytokines, as we observed a significant diminution of circulating IL-1 $\beta$ in the probiotics groups. Interestingly, it has been demonstrated that L. helveticus R0052 and L. rhamnosus R0011 prevent stress-induced memory deficits in mice infected by pathogens $^{(35)}$, reinforcing the immune-gut-brain axis hypothesis.

The intestinal epithelium forms a barrier between the external environment and luminal contents in which tight junction complexes have an important regulatory role ${ }^{(36)}$. Exposure to pro-inflammatory cytokines, such as TNF- $\alpha$, decreases epithelial barrier function and may thus lead to translocation of the bacterial component to the bloodstream. The present results indicate that intestinal barrier permeability may be involved, as we found it to be altered 2 weeks post-MI. As observed in chronic heart failure ${ }^{(37)}$, increased intestinal permeability has been documented and said to contribute to chronic inflammation in this pathological condition. Intestinal dysfunction may add to endotoxin translocation and ultimately augment blood lipopolysaccharide concentrations. Interestingly, normal volunteers injected with lipopolysaccharide exhibited acute increases in depression and anxiety symptoms, and their severity correlated with augmented peripheral blood cytokine concentrations ${ }^{(38)}$.

The presence of probiotics has a beneficial effect on intestinal barrier integrity through NF-кB pathway inhibition and intact extracellular signal-regulated kinases 1 and 2 signalling ${ }^{(23)}$. Another explanation may be related to the anti-apoptotic effect observed with these probiotics ${ }^{(16)}$, although this has not been demonstrated in intestinal tissue. Indeed, it has been determined that apoptosis affects intestinal barrier integrity ${ }^{(39)}$, and inhibition of apoptosis, through reduction of a cytokine-dependent mechanism, could be beneficial to intestinal integrity. The activation of toll-like receptors, a class of transmembrane pattern-recognition receptors that have a key role in microbial recognition, is 
another hypothesis that could explain the beneficial effect of probiotics on intestinal barrier integrity ${ }^{(40)}$. Probiotics activate toll-like receptor, such as toll-like receptor-2, and prevent intestinal barrier impairment, indicating a link between these events $^{(29,41)}$. Additional studies are needed to determine the mechanism by which $L$. helveticus and B. longum may be advantageous for intestinal barrier integrity.

Another possibility that could explain the beneficial effect of $L$. helveticus and $B$. longum on post-MI depression-like symptoms could be related to the perturbations that these probiotics may induce in the intestinal microbiota. According to the results obtained by Heijtz et al. ${ }^{(42)}$, microbial colonisation process initates signalling mechanisms that may affect the anxiety behaviour. Moreover, it has been observed that altered enteric flora induced by stress are responsible for colonic pathophysiology, and probiotics improve gut dysfunction by a mechanism that could involve the normalisation of the hypothalamic-pituitary-adrenal axis activity ${ }^{(43)}$. However, as long as we ignore the effect of MI on the intestinal microbiota, this will remain hypothetical.

In conclusion, the present study provided the first evidence of a positive outcome of probiotics combination (L. helveticus R0052 and B. longum R0175) on post-MI depression. The beneficial impact of probiotics combination also includes the maintenance of intestinal barrier integrity, which may contribute to the inflammatory state observed after MI.

\section{Limitations}

One concern is the possible physical consequences of thoracic surgery and MI itself in the capacity of rats to perform physically demanding behavioural tasks. We have previously shown, however, that sham and MI rats, having undergone the same thoracic surgery as reported here, performed similarly in the Morris water maze task, a test of motor performance and spatial memory requiring an intact hippocampus ${ }^{(11,44)}$, whether they were treated with the anti-depressant, desipramine, or saline, and improved with desipramine on the forced swimming test. The present results also indicated that, for similar myocardial infarct size, probiotics-treated MI rats were as efficient as sham controls on motor output measures such as swimming and escape time in the forced swimming test, which suggests that the level of injury was not sufficient to interfere with the results. Another concern is that more inflammatory cytokines should be analysed in order to support the notion according to which anti-inflammatory mechanisms underly the behavioural effects of probiotics reported here

\section{Acknowledgements}

The authors thank Melanie Gareau for her help in the measurement of intestinal barrier integrity. The present study was supported by grants from the Natural Sciences and Engineering Research Council of Canada (no. 250234-07). Institut Rosell-Lallemand provided the probiotics administered in these experiments. J. A. B., I. R., K. G., S.-A. G. and T. A. T. contributed to the experiments, data analysis and writing, and R. G. and G. R. contributed to conception of the experiments, data analysis and writing.

\section{References}

1. Guck TP, Kavan MG, Elsasser GN, et al. (2001) Assessment and treatment of depression following myocardial infarction. Am Fam Physician 64, 641-648.

2. Meneses R, Almeida MC, Abecasis J, et al. (2007) Depression in patients with myocardial infarction. Rev Port Cardiol 26, $1143-1165$

3. Bush DE, Ziegelstein RC, Tayback M, et al. (2001) Even minimal symptoms of depression increase mortality risk after acute myocardial infarction. Am J Cardiol 88, 337-341.

4. Lesperance F, Frasure-Smith N, Talajic M, et al. (2002) Fiveyear risk of cardiac mortality in relation to initial severity and one-year changes in depression symptoms after myocardial infarction. Circulation 105, 1049-1053.

5. Frasure-Smith N, Lesperance F \& Talajic M (1993) Depression following myocardial infarction. Impact on 6-month survival. JAMA 270, 1819-1825.

6. Frasure-Smith N, Lesperance F \& Talajic M (1995) The impact of negative emotions on prognosis following myocardial infarction: is it more than depression? Health Psychol 14, 388-398.

7. Raison CL, Capuron L \& Miller AH (2006) Cytokines sing the blues: inflammation and the pathogenesis of depression. Trends Immunol 27, 24-31.

8. Kaloustian S, Bah TM, Rondeau I, et al. (2009) Tumor necrosis factor-alpha participates in apoptosis in the limbic system after myocardial infarction. Apoptosis 14, 1308-1316.

9. Francis J, Chu Y, Johnson AK, et al. (2004) Acute myocardial infarction induces hypothalamic cytokine synthesis. Am J Physiol 286, H2264-H2271.

10. Koo JW \& Duman RS (2009) Evidence for IL-1 receptor blockade as a therapeutic strategy for the treatment of depression. Curr Opin Investig Drugs 10, 664-671.

11. Wann BP, Bah TM, Boucher M, et al. (2007) Vulnerability for apoptosis in the limbic system after myocardial infarction in rats: a possible model for human postinfarct major depression. J Psychiatry Neurosci 32, 11-16.

12. Wann BP, Bah TM, Kaloustian S, et al. (2009) Behavioural signs of depression and apoptosis in the limbic system following myocardial infarction: effects of sertraline. J Psychopharmacol 23, 451-459.

13. Wann BP, Boucher M, Kaloustian S, et al. (2006) Apoptosis detected in the amygdala following myocardial infarction in the rat. Biol Psychiatry 59, 430-433.

14. Kaloustian S, Wann BP, Bah TM, et al. (2008) Apoptosis time course in the limbic system after myocardial infarction in the rat. Brain Res 1216, 87-91.

15. Kaloustian S, Wann BP, Bah TM, et al. (2007) Celecoxib after the onset of reperfusion reduces apoptosis in the amygdala. Apoptosis 12, 1945-1951.

16. Girard SA, Bah TM, Kaloustian S, et al. (2009) Lactobacillus helveticus and Bifidobacterium longum taken in combination reduce the apoptosis propensity in the limbic system after myocardial infarction in a rat model. Br J Nutr 102, 1420-1425.

17. Cazzola M, Tompkins TA \& Matera MG (2010) Immunomodulatory impact of a synbiotic in T(h)1 and T(h)2 models of infection. Ther Adv Respir Dis 4, 259-270.

18. Wagar LE, Champagne CP, Buckley ND, et al. (2009) Immunomodulatory properties of fermented soy and dairy 
milks prepared with lactic acid bacteria. J Food Sci $\mathbf{7 4}$, M423-M430.

19. Bah TM, Benderdour M, Kaloustian S, et al. (2011) Escitalopram reduces circulating pro-inflammatory cytokines and improves depressive behavior without affecting sleep in a rat model of post-cardiac infarct depression. Behav Brain Res 225, 243-251.

20. Diop L, Guillou S \& Durand H (2008) Probiotic food supplement reduces stress-induced gastrointestinal symptoms in volunteers: a double-blind, placebo-controlled, randomized trial. Nutr Res 28, 1-5.

21. Messaoudi M, Lalonde R, Violle N, et al. (2011) Assessment of psychotropic-like properties of a probiotic formulation (Lactobacillus belveticus R0052 and Bifidobacterium longum R0175) in rats and human subjects. Br J Nutr 105, 755-764.

22. Wann BP, Béland SG, Rousseau G, et al. (2004) Permeability in the limbic system following myocardial infarction in the rat. In Society for Neuroscience 34th Annual Meeting, \#568.4.

23. Donato KA, Gareau MG, Wang YJ, et al. (2010) Lactobacillus rhamnosus GG attenuates interferon-\{gamma\} and tumor necrosis factor-\{alpha\}-induced barrier dysfunction and proinflammatory signalling. Microbiology 156, 3288-3297.

24. Catalioto RM, Maggi CA \& Giuliani S (2011) Intestinal epithelial barrier dysfunction in disease and possible therapeutical interventions. Curr Med Chem 18, 398-426.

25. Johnson-Henry KC, Hagen KE, Gordonpour M, et al. (2007) Surface-layer protein extracts from Lactobacillus belveticus inhibit enterohaemorrhagic Escherichia coli O157:H7 adhesion to epithelial cells. Cell Microbiol 9, 356-367.

26. Sherman PM, Johnson-Henry KC, Yeung HP, et al. (2005) Probiotics reduce enterohemorrhagic Escherichia coli O157:H7- and enteropathogenic E. coli O127:H6-induced changes in polarized T84 epithelial cell monolayers by reducing bacterial adhesion and cytoskeletal rearrangements. Infect Immun 73, 5183-5188.

27. Lammers KM, Brigidi P, Vitali B, et al. (2003) Immunomodulatory effects of probiotic bacteria DNA: IL-1 and IL-10 response in human peripheral blood mononuclear cells. FEMS Immunol Med Microbiol 38, 165-172.

28. Yan F \& Polk DB (2002) Probiotic bacterium prevents cytokine-induced apoptosis in intestinal epithelial cells. J Biol Chem 277, 50959-50965.

29. Eun CS, Kim YS, Han DS, et al. (2011) Lactobacillus casei prevents impaired barrier function in intestinal epithelial cells. APMIS 119, 49-56.
30. Fields A (2009) Discovering statistics using SPSS. In Discovering Statistics using SPSS, 3rd ed., p. 822. London: SAGE Publications Ltd.

31. Dantzer R (2001) Cytokine-induced sickness behavior: where do we stand? Brain Behav Immun 15, 7-24.

32. Kent S, Bluthé RM, Kelley KW, et al. (1992) Sickness behavior as a new target for drug development. Trends Pharmacol Sci 13, 24-28.

33. Besedovsky HO, del Rey A, Sorkin E, et al. (1986) Immunoregulatory feedback between interleukin-1 and glucocorticoid hormones. Science 233, 652-654.

34. Castanon N, Leonard BE, Neveu PJ, et al. (2002) Effects of antidepressants on cytokine production and actions. Brain Behav Immun 16, 569-574.

35. Gareau MG, Wine E, Rodrigues DM, et al. (2011) Bacterial infection causes stress-induced memory dysfunction in mice. Gut 60, 307-317.

36. Cario E (2008) Barrier-protective function of intestinal epithelial toll-like receptor 2. Mucosal Immunol 1, Suppl. 1, S62-S66.

37. Sandek A, Bauditz J, Swidsinski A, et al. (2007) Altered intestinal function in patients with chronic heart failure. J Am Coll Cardiol 50, 1561-1569.

38. Yirmiya R, Pollak Y, Morag M, et al. (2000) Illness, cytokines, and depression. Ann N Y Acad Sci USA 917, 478-487.

39. Bruewer M, Luegering A, Kucharzik T, et al. (2003) Proinflammatory cytokines disrupt epithelial barrier function by apoptosis-independent mechanisms. J Immunol 171, 6164-6172.

40. Cario E, Gerken G \& Podolsky DK (2007) Toll-like receptor 2 controls mucosal inflammation by regulating epithelial barrier function. Gastroenterology 132, 1359-1374.

41. Karczewski J, Troost FJ, Konings I, et al. (2010) Regulation of human epithelial tight junction proteins by Lactobacillus plantarum in vivo and protective effects on the epithelial barrier. Am J Physiol Gastrointest Liver Physiol 298, G851-G859.

42. Heijtz RD, Wang S, Anuar F, et al. (2011) Normal gut microbiota modulates brain development and behavior. Proc Natl Acad Sci U S A 108, 3047-3052.

43. Gareau MG, Jury J, MacQueen G, et al. (2007) Probiotic treatment of rat pups normalises corticosterone release and ameliorates colonic dysfunction induced by maternal separation. Gut 56, 1522-1528.

44. Morris R (1984) Developments of a water-maze procedure for studying spatial learning in the rat. J Neurosci Methods 11, $47-60$. 